\title{
Values of \\ Domination Numbers of the Queen's Graph
}

\author{
Patric R. J. Östergård* \\ Department of Computer Science and Engineering \\ Helsinki University of Technology \\ P.O. Box 5400 \\ 02015 HUT, Finland \\ patric.ostergard@hut.fi \\ William D. Weakley \\ Department of Mathematical Sciences \\ Indiana University - Purdue University Fort Wayne \\ Fort Wayne, Indiana 46805 \\ weakley@ipfw.edu
}

Submitted: November 7, 2000; Accepted: March 26, 2001.

MR Subject Classifications: 05C69, 68R05

\begin{abstract}
The queen's graph $Q_{n}$ has the squares of the $n \times n$ chessboard as its vertices; two squares are adjacent if they are in the same row, column, or diagonal. Let $\gamma\left(Q_{n}\right)$ and $i\left(Q_{n}\right)$ be the minimum sizes of a dominating set and an independent dominating set of $Q_{n}$, respectively. Recent results, the Parallelogram Law, and a search algorithm adapted from Knuth are used to find dominating sets. New values and bounds:

(A) $\gamma\left(Q_{n}\right)=\lceil n / 2\rceil$ is shown for 17 values of $n$ (in particular, the set of values for which the conjecture $\gamma\left(Q_{4 k+1}\right)=2 k+1$ is known to hold is extended to $k \leq 32$ );

(B) $i\left(Q_{n}\right)=\lceil n / 2\rceil$ is shown for 11 values of $n$, including 5 of those from (A);

(C) One or both of $\gamma\left(Q_{n}\right)$ and $i\left(Q_{n}\right)$ is shown to lie in $\{\lceil n / 2\rceil,\lceil n / 2\rceil+1\}$ for 85 values of $n$ distinct from those in (A) and (B).

Combined with previously published work, these results imply that for $n \leq 120$, each of $\gamma\left(Q_{n}\right)$ and $i\left(Q_{n}\right)$ is either known, or known to have one of two values.

Also, the general bounds $\gamma\left(Q_{n}\right) \leq 69 n / 133+O(1)$ and $i\left(Q_{n}\right) \leq 61 n / 111+O(1)$ are established.
\end{abstract}

Keywords: dominating set, queen domination, queen's graph.

${ }^{*}$ Supported by the Academy of Finland. 


\section{Introduction}

The queen's graph $Q_{n}$ has the squares of the $n \times n$ chessboard as its vertices; two squares are adjacent if they are in the same row, column, or diagonal. A set $D$ of squares of $Q_{n}$ is a dominating set for $Q_{n}$ if every square of $Q_{n}$ is either in $D$ or adjacent to a square in $D$. If no two squares of a set $I$ are adjacent then $I$ is an independent set. Let $\gamma\left(Q_{n}\right)$ denote the minimum size of a dominating set for $Q_{n}$; a dominating set of this size is a minimum dominating set. Let $i\left(Q_{n}\right)$ denote the minimum size of an independent dominating set for $Q_{n}$.

The problems of finding values of $\gamma\left(Q_{n}\right)$ and of $i\left(Q_{n}\right)$ are given as Problem C18 in [10], and have interested mathematicians for well over a century. De Jaenisch [13] considered these problems in 1862. In 1892, Rouse Ball [16] gave dominating sets and independent dominating sets of $Q_{n}$ for $n \leq 8$. Ahrens [1] extended this in 1910 to $n \leq 13$ and $n=17$ for $\gamma\left(Q_{n}\right)$ and to $n \leq 12$ for $i\left(Q_{n}\right)$. In most cases, proof that these sets were minimum dominating sets had to wait for recent work on lower bounds.

Spencer proved $[7,17]$ that

$$
\gamma\left(Q_{n}\right) \geq(n-1) / 2
$$

The only values for which equality is known to hold in (1) are $n=3,11$, so researchers have sought better bounds. Weakley showed [17] that

$$
\gamma\left(Q_{4 k+1}\right) \geq 2 k+1
$$

and that $i\left(Q_{4 k+1}\right)=\gamma\left(Q_{4 k+1}\right)=2 k+1$ for $k \leq 6$ and $k=8$. Other researchers [2, 5, 9] have gone further, showing $\gamma\left(Q_{4 k+1}\right)=2 k+1$ for $k \leq 15$ and $k=17,19$. Later we extend this to $k \leq 32$.

Burger and Mynhardt showed [3] that $\gamma\left(Q_{4 k+3}\right) \geq 2 k+2$ for $3 \leq k \leq 7$, with equality for $k=4,7$. Weakley proved [19] the following theorem.

Theorem 1 Define a sequence of integers by $n_{1}=3, n_{2}=11$, and $n_{i}=4 n_{i-1}-n_{i-2}-2$ for $i>2$. If $\gamma\left(Q_{n}\right)=(n-1) / 2$ then $n=n_{i}$ for some $i$.

The first four values in the sequence of Theorem 1 are $3,11,39,143$. As $\gamma\left(Q_{39}\right)=20$ was shown in [19, Proposition 7], Theorem 1 and (1) imply the following bound.

Corollary 2 If $n<143$ and $n \neq 3,11$, then $\gamma\left(Q_{n}\right) \geq n / 2$.

In this paper, we employ dominating sets called $p$-covers (defined below), with slight variations. A $p$-cover of $Q_{n}$ is required to occupy certain lines (rows, columns, diagonals), and also occupies a few other lines if its size exceeds $(n-1) / 2$. For these other lines, the Parallelogram Law (Theorem 4 below) allows us to greatly restrict the possibilities that need be considered. For $n \leq 35$, straightforward hand calculation suffices to either find corresponding dominating sets, or show that none exists. For larger $n$, we use computer search with the algorithm described in Section 4 . The resulting dominating sets are given 
in Section 5, as are the values and bounds for $\gamma\left(Q_{n}\right)$ and $i\left(Q_{n}\right)$ implied by the sets and Corollary 2.

We devote considerable effort to proving for various $n$ that one or both of $\gamma\left(Q_{n}\right), i\left(Q_{n}\right)$ lies in $\{\lceil n / 2\rceil,\lceil n / 2\rceil+1\}$. Of course, exact values would be preferable. However, it is our belief that often $\lceil n / 2\rceil+1$ is correct, but that it will be quite difficult to prove that $\lceil n / 2\rceil$ cannot be achieved. For example, current knowledge that $\gamma\left(Q_{n}\right)>\lceil n / 2\rceil$ for $n=8,14-16$ and that $i\left(Q_{n}\right)>\lceil n / 2\rceil$ for $n=4,6,8,12,14-16$ comes from exhaustive search, which is not feasible for large $n$.

Also, we use dominating sets found for $Q_{131}$ and $Q_{109}$ and work from [18] to give improved upper bounds for $\gamma\left(Q_{n}\right)$ and $i\left(Q_{n}\right)$.

\section{Preliminaries}

For odd positive integers $n$, we will identify the $n \times n$ chessboard with a square of side length $n$ in the Cartesian plane, having sides parallel to the coordinate axes. We place the board with its center at the origin of the coordinate system, and refer to board squares by the coordinates of their centers. The square $(x, y)$ is in column $x$ and row $y$. Columns and rows will be referred to collectively as orthogonals. The difference diagonal (respectively sum diagonal) through square $(x, y)$ is the set of all board squares with centers on the line of slope +1 (respectively -1 ) through the point $(x, y)$. The value of $y-x$ is the same for each square $(x, y)$ on a difference diagonal, and we will refer to the diagonal by this value. Similarly, the value of $x+y$ is the same for each square on a sum diagonal, and we associate this value to the diagonal. The long diagonals of $Q_{n}$ are difference diagonal 0 and sum diagonal 0 .

For even $n$, we obtain $Q_{n}$ by adding a row and column to $Q_{n-1}$.

The square $(x, y)$ is even if $x+y$ is even, odd if $x+y$ is odd. We divide the even squares of $Q_{n}$ into two classes: $(x, y)$ is even-even if both $x$ and $y$ are even, odd-odd if both are odd.

We now describe the dominating sets which have proven most useful in recent work $[2,3,4,5,6,8,9,14,17,18,19]$ on queen domination.

Definitions. Let $n$ be an odd positive integer, let $D$ be a set of squares of $Q_{n}$, and let $p \in\{0,1\}$. Say that $D$ is a $p$-orthodox set if every orthogonal of parity $p$ contains a square of $D$.

If $D$ is a 0 -orthodox set and every odd-odd square of $Q_{n}$ shares a diagonal with some square of $D$, we will say that $D$ is a 0 -cover. If $D$ is a 1 -orthodox set and every even-even square shares a diagonal with some square of $D$, we say $D$ is a 1 -cover.

It is clear from the definition that a $p$-cover dominates every even square, and every odd square is on one orthogonal of parity $p$, so is also covered: a $p$-cover is a dominating set.

Definitions. Let $n$ be an odd positive integer and suppose that $D$ is a set of squares of $Q_{n}$ containing a square of each long diagonal. 
Define $e=e(D)$ to be the largest integer such that for each $i$ with $|i| \leq e, D$ contains a square of difference diagonal $2 i$.

Define $f=f(D)$ to be the largest integer such that for each $i$ with $|i| \leq f, D$ contains a square of sum diagonal $2 i$.

Define $u=u(D)$ to be the largest integer such that for each $i$ with $1 \leq i \leq u, D$ contains squares of difference diagonals $\pm(2 e+4 i)$ and squares of sum diagonals $\pm(2 f+4 i)$.

The following characterization of $p$-covers was proved in [18].

Theorem 3 Let $n$ be an odd positive integer and let $p \in\{0,1\}$. Let $D$ be a p-orthodox set for $Q_{n}$ that contains at least one square from each of the long diagonals, and let $e=e(D)$, $f=f(D)$, and $u=u(D)$. The following are equivalent:

(1) $D$ is a p-cover of $Q_{n}$;

(2) Either $(A) e+f \equiv p(\bmod 2)$ and $e+f+2 u \geq(n-5) / 2$,

or $\quad(B) e+f \equiv 1-p(\bmod 2)$ and $e+f \geq(n-3) / 2$.

Definition. We say a $p$-cover is type $A$ or type $B$ depending on whether it satisfies condition $(2 \mathrm{~A})$ or $(2 \mathrm{~B})$ of Theorem 3 .

Type A 0-covers have been used $[6,8,17]$ to produce upper bounds for $\gamma\left(Q_{n}\right)$ and $i\left(Q_{n}\right)$. Also, most of the work $[2,5,9,14,17]$ done to establish $\gamma\left(Q_{4 k+1}\right)=2 k+1$ for $k \leq 15$ and $k=17,19$ used type A $p$-covers.

Type B $p$-covers are denser central packings than type A, and are less useful for producing small dominating sets, so we only use type A.

We require the following theorem from [19].

Theorem 4 (Parallelogram Law) Let $S$ be a set of $k$ squares of $Q_{n}$ occupying columns numbered $\left(x_{i}\right)_{i=1}^{k}$, rows $\left(y_{i}\right)_{i=1}^{k}$, difference diagonals $\left(d_{i}\right)_{i=1}^{k}$, and sum diagonals $\left(s_{i}\right)_{i=1}^{k}$. Then

$$
2 \sum_{i=1}^{k} x_{i}^{2}+2 \sum_{i=1}^{k} y_{i}^{2}=\sum_{i=1}^{k} d_{i}^{2}+\sum_{i=1}^{k} s_{i}^{2} .
$$

Our general upper bounds derive from the next theorem, which was proved in [18].

Theorem 5 Let $n$ be an odd positive integer, let $p \in\{0,1\}$, and let $D$ be a type $A$-cover of $Q_{n}$ that contains d squares, including a square of each long diagonal.

If $p=0$ and $n \equiv 1(\bmod 4)$, or $p=1$ and $n \equiv-1(\bmod 4)$, then for all $k, \gamma\left(Q_{k}\right) \leq$ $\frac{d+3}{n+2} k+O(1)$; if also $D$ is independent, then for all $k, i\left(Q_{k}\right) \leq \frac{d+6}{n+2} k+O(1)$.

If $p=1$ and $n \equiv 1(\bmod 4)$, or $p=0$ and $n \equiv-1(\bmod 4)$, and $D$ contains no edge squares, then for all $k, \gamma\left(Q_{k}\right) \leq \frac{d+1}{n} k+O(1)$; if also $D$ is independent, then for all $k$, $i\left(Q_{k}\right) \leq \frac{d+2}{n} k+O(1)$. 
Below we will give a type A 1-cover of size 66 for $Q_{131}$ and an independent type A 0-cover of size 55 for $Q_{109}$. By Theorem 5 , these imply the following bounds.

Corollary $6 \gamma\left(Q_{n}\right) \leq 69 n / 133+O(1)$ and $i\left(Q_{n}\right) \leq 61 n / 111+O(1)$

The best previously published upper bound for $\gamma\left(Q_{n}\right)$ is $8 n / 15+O(1)$ from [4], and $69 / 133$ is about $43 \%$ of the way from $8 / 15$ to the $1 / 2$ of the lower bound (1). The best previously published upper bound for $i\left(Q_{n}\right)$ is $19 n / 33+O(1)$ from [18], and 61/111 is about $34 \%$ of the way from $19 / 33$ to $1 / 2$.

\section{Constructions}

Our constructions are all on $Q_{n}$ with $n$ odd; the long diagonals are occupied, so $e$ and $f$ are defined. By rotating the board if necessary, we will always take $e \geq f$. All of our dominating sets have size in $\{\lceil n / 2\rceil,\lceil n / 2\rceil+1\}$, and all but one are type A $p$-covers. The following generalizes Theorem 3 of [5].

Proposition 7 Let $D$ be a type $A$-cover of $Q_{n}$, with $(n+1) / 2$ members. If $p=0$, then either $e=f$ or $e=f+2$. If $p=1$, then $e=f+1$.

Proof. Counting occupied difference diagonals, we have $2 e+1+2 u \leq(n+1) / 2$. Subtracting this from inequality (2A) of Theorem 3 gives $e-f \leq 2$. Then $e \geq f$ and $e+f \equiv p(\bmod 2)$ imply the conclusion.

For each construction we use, there are specific lines that must be occupied; we will refer to these as required lines, and to other lines occupied by the dominating set as excess lines. Complicating the picture slightly is the fact that a line may be occupied $m$ times, with $m>1$. If such a line is required, we also regard it as an excess line occurring $m-1$ times.

Although the excess lines do not contribute to domination, their values are strongly restricted, both by the linear constraints

$$
\left(\sum y_{i}\right)-\left(\sum x_{i}\right)=\sum\left(y_{i}-x_{i}\right) \text { and }\left(\sum y_{i}\right)+\left(\sum x_{i}\right)=\sum\left(y_{i}+x_{i}\right)
$$

and by the quadratic constraint due to the Parallelogram Law.

We now describe four constructions that gave us exact values of $\gamma\left(Q_{n}\right)$ or $i\left(Q_{n}\right)$; the first two constructions generalize those of [5]. As all the constructions are similar, we go into detail only for the first, which gave more exact values than the others. The constructions we used to establish bounds of the type $\gamma\left(Q_{n}\right)$ or $i\left(Q_{n}\right)$ in $\{\lceil n / 2\rceil,\lceil n / 2\rceil+1\}$ are also like the ones described here.

Type A 0-cover of size $2 k+1$ for $Q_{4 k+1}$ with $e=f+2$.

Here the required row numbers and column numbers are the members of $\{2 j:-k \leq j \leq$ $k\}$; as there are $2 k+1$ of these, we have no excess rows or columns. From $e=f+2$, $n=4 k+1$, and $(2 \mathrm{~A})$ of Theorem 3 , we see $u \geq k-e$. The number of required difference 
diagonals is at most the size $2 k+1$ of the dominating set, so $2 e+1+2 u \leq 2 k+1$, which implies that $u=k-e$ and that there are no excess difference diagonals. Then the number of required sum diagonals is $2 f+1+2 u=2 k-3$, so there are four excess sum diagonals, which we will denote by $\left(s_{i}\right)_{i=1}^{4}$.

Let $D=\left\{\left(x_{i}, y_{i}\right)\right\}_{i=1}^{2 k+1}$ be our 0 -cover, with the squares numbered so that excess sum diagonal $s_{i}$ is occupied by $\left(x_{i}, y_{i}\right)$ for $1 \leq i \leq 4$. As the required sum diagonals other than 0 come in pairs with sum 0 , we have $\sum_{i>4}\left(y_{i}+x_{i}\right)=0$, so $\sum_{i \geq 1}\left(y_{i}+x_{i}\right)=\sum_{i=1}^{4} s_{i}$. Looking at the required orthogonal numbers, we see $\sum_{i \geq 1} x_{i}=\sum_{i \geq 1} y_{i}=0$, so $\sum_{i \geq 1}\left(y_{i}+x_{i}\right)=0$ and thus

$$
s_{1}+s_{2}+s_{3}+s_{4}=0 .
$$

The Parallelogram Law implies

$$
\begin{aligned}
2\left(2 \sum_{i=1}^{k}(2 i)^{2}\right)+ & 2\left(2 \sum_{i=1}^{k}(2 i)^{2}\right)=\left(2 \sum_{i=1}^{e}(2 i)^{2}+2 \sum_{i=1}^{u}(2 e+4 i)^{2}\right)+ \\
& \left(s_{1}^{2}+s_{2}^{2}+s_{3}^{2}+s_{4}^{2}+2 \sum_{i=1}^{f}(2 i)^{2}+2 \sum_{i=1}^{u}(2 f+4 i)^{2}\right)
\end{aligned}
$$

and using $f=e-2$ and $u=k-e$, we can simplify this to

$$
s_{1}^{2}+s_{2}^{2}+s_{3}^{2}+s_{4}^{2}=8-8(2 k-1)\left[e^{2}-(2 k+1) e+2 k(k-1) / 3\right] .
$$

For each $k \leq 32$, we found all values of $e$ and sequences $\left(s_{i}\right)_{i=1}^{4}$ satisfying (4) and (5). (This can easily be extended to larger $k$, but the remaining problem of finding $D$ then has too large a search space for our approach.) It is interesting that for each $k$, e must be near $\left(1-\frac{1}{\sqrt{3}}\right) k$; we sketch a proof.

Let $g(k, e)$ denote the right side of $(5)$. From $(5)$, we see that $g(k, e) \geq 0$, and then that the form of $g(k, e)$ implies $g(k, e) \geq 8$, so $e^{2}-(2 k+1) e+2 k(k-1) / 3 \leq 0$. This implies

$$
e \geq e_{\min }=k+0.5-\sqrt{\left[(k+2.5)^{2}-5.5\right] / 3}>\left(1-\frac{1}{\sqrt{3}}\right) k-\frac{5-\sqrt{3}}{2 \sqrt{3}} .
$$

Since no row, column, or difference diagonal can contain more than one square of $D$, (5) implies $(4 k)^{2}+2(4 k-2)^{2}+(4 k-6)^{2} \geq g(k, e)$. For $k>5$, this gives

$$
e \leq e_{\max }=k+0.5-\sqrt{\left[(k-3.5)^{2}-4\right] / 3},
$$

and also $e_{\max }-e_{\min }<2 \sqrt{3} \approx 3.46$. Thus there are at most four values of $e$ satisfying (4) and (5), all near $\left(1-\frac{1}{\sqrt{3}}\right) k$. Quite similar bounds can be proved for the other constructions below.

The search method described in Section 4 allowed us to find the dominating sets for $k=$ $6,11,14,17-20,22-24,27,29-32$ given later. If the excess sum diagonals are all distinct and different from the required sum diagonals, as with those given for $k=6,11,14,17-20$, 22-24, 27, the resulting dominating set is independent.

As we show next, if the excess sum diagonals include certain values, we get information about $Q_{4 k+2}$ and possibly $Q_{4 k+3}$. 
Proposition 8 Suppose there is a 0-cover $D$ of size $2 k+1$ for $Q_{4 k+1}$ with $e=f+2$. (Thus $\gamma\left(Q_{4 k+1}\right)=2 k+1$.) Let $S$ be the set of numbers of excess sum diagonals of $D$, and let $L=\{ \pm(2 f+2), \pm(4 k-2 f-4)\}$.

(A) If $S \cap L$ has two members with the same sign, then $\gamma\left(Q_{4 k+2}\right)=2 k+1$. If also $D$ is independent, then $i\left(Q_{4 k+2}\right)=2 k+1$.

(B) If $|S \cap L| \geq 1$ and $D$ is independent, then $i\left(Q_{4 k+2}\right) \in\{2 k+1,2 k+2\}$.

(C) If $|S \cap L| \geq 2$ and $D$ is independent, then $i\left(Q_{4 k+3}\right) \in\{2 k+2,2 k+3\}$.

Proof. Two ways to obtain a copy of $Q_{4 k+2}$ from $Q_{4 k+1}$ are by adjoining either row and column $2 k+1$ of $Q_{4 k+3}$ or row and column - $(2 k+1)$ of $Q_{4 k+3}$; adjoining all of these gives $Q_{4 k+3}$. We ask which squares of these orthogonals are not covered by the required lines for a 0 -cover $D$ of size $2 k+1$ for $Q_{4 k+1}$ with $e=f+2$; since the set of required lines of $D$ is symmetric across each of the long diagonals, it suffices to examine row $2 k+1$. Suppose square $s=(x, 2 k+1)$ is a square of $Q_{4 k+3}$ not covered by $D$.

Since $|x| \leq 2 k+1$, if $x$ is even then column $x$ is occupied by $D$, so we may conclude $s$ is an odd-odd square, and thus lies on an empty difference diagonal with even number (say) $m$.

If $m \equiv 2 e(\bmod 4)$, then by the definition of $e$ and $u$, we have $m=2 e+4 u+4+4 i$ for some $i \geq 0$. Above it was shown that $u=k-e$, and we are assuming that $f=e-2$, implying that $s$ lies on sum diagonal $2 f+2-4 i$, which is a required diagonal unless $i=0$. Thus $s$ is on sum diagonal $2 f+2$ in this case.

Otherwise $m \equiv 2 e+2(\bmod 4)$, so $m=2 e+2+4 i$ for some $i \geq 0$, and a similar argument shows that $s$ lies on sum diagonal $4 k-2 f-4$.

Thus if the excess sum diagonals of $D$ include $2 f+2$ and $4 k-2 f-4$, then $D$ covers row and column $2 k+1$ and therefore dominates a copy of $Q_{4 k+2}$; likewise if $-(2 f+2)$ and $-(4 k-2 f-4)$ are excess sum diagonals of $D$. This establishes (A).

Now suppose $D$ is independent and $S$ contains (say) one but not both of sum diagonals $2 f+2$ and $4 k-2 f-4$. Then the other of these meets row $2 k+1$ in a square $D$ does not cover, and adding this square to $D$ gives an independent dominating set of $Q_{4 k+2}$. The rest is similar.

Type A 0-cover of size $2 k+1$ for $Q_{4 k+1}$ with $e=f$.

The required row and column numbers are as in the previous case, so again there are no excess rows or columns. By (2A) of Theorem 3, the minimum value of $u$ for a 0 -cover is $u=k-e-1$; then for each kind of diagonal, there are $2 e+1+2 u=2 k-1$ required values, leaving excess difference diagonals $d_{1}, d_{2}$ and excess sum diagonals $s_{1}, s_{2}$. The linear constraints (3) give $d_{1}+d_{2}=0$ and $s_{1}+s_{2}=0$. The Parallelogram Law gives

$$
d_{1}^{2}+s_{1}^{2}=-4\left[(2 k-1) e^{2}-(2 k-1)^{2} e+2 k\left(2 k^{2}-9 k+1\right) / 3\right] .
$$

This construction is much less versatile than the previous one. It led to the dominating sets for $k=26,28$ given later, with the one for $k=26$ being independent. 
Type A 1-cover of size $2 k+1$ for $Q_{4 k+1}$ with $e=f+1$.

Here the required row and column numbers are $\pm 1, \pm 3, \ldots, \pm(2 k-1)$, so there is an excess column $a$ and an excess column $b$. By (2A) of Theorem 3, the minimum possible value of $u$ is $k-e$, and then there are no excess difference diagonals and two excess sum diagonals, say $s_{1}$ and $s_{2}$. From (3), we have $b-a=\sum y_{i}-\sum x_{i}=\sum\left(y_{i}-x_{i}\right)=0$, so $a=b$, and also $s_{1}+s_{2}=2 a$. From the Parallelogram Law,

$$
s_{1}^{2}+s_{2}^{2}-4 a^{2}=-16\left[e^{2}-(2 k+1) e+(k+1)(2 k+1) / 3\right] .
$$

This method produced dominating sets for $k=1-11,14,15,17,19-22,25-27$. Of these, only the set for $k=21$ gives a value not supplied by the previous constructions. However, if $|a| \neq 2 k$, as with all of ours, these sets have no squares in edge rows or columns, thus giving information about $Q_{4 k}$ and $Q_{4 k-1}$. For $k=21,25,26$ we get new bounds.

Type A 1-cover of size $2 k+2$ for $Q_{4 k+3}$ with $e=f+1$.

The required row and column numbers are $\pm 1, \pm 3, \ldots, \pm(2 k-1)$, so there are no excess rows or columns. By Theorem $3(2 \mathrm{~A})$, the minimum possible value of $u$ is $k-e$, which gives an excess difference diagonal $d_{1}$ and excess sum diagonals $s_{1}, s_{2}, s_{3}$. Then (3) implies $d_{1}=0$, so dominating sets of this kind are not independent, and $s_{1}+s_{2}+s_{3}=0$. The Parallelogram Law gives

$$
s_{1}^{2}+s_{1} s_{2}+s_{2}^{2}=4-8 k\left[e^{2}-(2 k+1) e+(k+1)(2 k-5) / 3\right] .
$$

This yielded dominating sets for $k=1,2,4,5,7$, confirming values already known, and also for new values $k=17,22,28,32$, as given later.

\section{The Search Algorithm}

We shall here describe a computer search for covers discussed in the previous section. Once we have chosen the size of the board and the type of cover, the theory developed is first used to find admissible values of the parameter $e$ and positions of the excess lines. We shall first discuss the case of independent dominating sets, and then briefly look at the search for dependent dominating sets.

The precalculations give a set $L$ of the lines to be occupied; queens can only be placed in squares that occupy four such lines. These squares are called eligible. We now list all eligible squares and to each square $i$ associate the set $S_{i}$ of the lines that it occupies. For independent dominating sets, the computational problem is now to find a set of eligible squares whose sets $S_{i}$ partition $L$. This problem is known as the exact cover problem.

Knuth [15] has recently developed a very fast program for the exact cover problem. The program, which can be downloaded from Knuth's web page, in particular uses an idea of Hitotumatu and Noshita [12] for efficiently handling pointers in backtrack programs.

This approach is orders of magnitude faster than searching for dominating sets from scratch. Of course, the approach only works if there are dominating sets of the given types. The results of this work, however, are encouraging. We must remark, though, that 
for a given size of the board there are many parameter sets that can be tried, and we often had to try several of these before finding a solution (if any).

There is also a limit for this approach. For the smallest instances, a complete search only takes seconds. For somewhat larger instances, when a complete search is not feasible, one can hope that the first solution of the backtrack search is encountered within a reasonable time. We stopped the search at board sizes for which days of cpu time are needed to find a solution. (But note that although larger boards generally lead to longer computer runs, there is a big variation in the cpu time needed for different instances.)

If the parameter set admits, one can try to impose more structure on the dominating set, such as requiring a solution to have a $180^{\circ}$ rotational symmetry. This approach, which reduces the search space considerably, was successful only in a few cases.

If we search for a dominating set that is not independent, we have a problem slightly different from the exact cover problem. Fortunately, Knuth's program can be slightly modified to handle such instances also. Without going into details, the main idea is to associate a positive integer to each element in $L$ telling how many times the element must occur in the sets of a solution. These values are incremented and decremented during the search, and elements with value 1 are treated as in the original algorithm. Especially when all sets $S_{i}$ have at least one element with value 1, which is the case in all our instances, this modification is straightforward.

\section{The Dominating Sets}

Below are given the dominating sets that, along with Corollary 2, establish new values or bounds for $\gamma\left(Q_{n}\right)$ and $i\left(Q_{n}\right)$. Each description begins with the new bounds or values implied (other than those due to the elementary fact $\gamma\left(Q_{n+1}\right) \leq \gamma\left(Q_{n}\right)+1$ ); any previously published value that is implied is given parenthetically. The kind of dominating set is given (all $p$-covers used are type A), then the values of $e, f, u$, the excess lines, and finally the squares of the set.

$i\left(Q_{26}\right) \in\{13,14\}$ and $\gamma\left(Q_{27}\right)=14$ (and $\left.i\left(Q_{25}\right)=13\right)$ : begin with a 0-cover of size 13 for $Q_{25}$ with $e=3, f=1, u=3$ and excess sum diagonals $-22,-4,8,18$. For even $x$ from -12 to $12, y$-values are $6,-12,-6,8,0,-8,10,-2,4,12,-10,-4,2$. Ву Proposition $6(\mathrm{~B})$, adding the square $(-9,13)$ gives $i\left(Q_{26}\right) \in\{13,14\}$. If instead we add the square $(-9,-9)$, we obtain a 0 -orthodox set that misses being a 0 -cover of $Q_{27}$ with $e=f=u=3$ only by failing to occupy sum diagonal 4. However, every even-even square of sum diagonal 4 is orthogonally covered, and the only odd-odd squares of sum diagonal 4 not covered along their difference diagonals are $(-9,13)$ and $(13,-9)$, which are covered by $(-9,-9)$. Thus the set dominates $Q_{27}$.

$i\left(Q_{27}\right), i\left(Q_{28}\right) \in\{14,15\}$ : 1-cover of size 15 for $Q_{27}$ with $e=6, f=5, u=0$, excess difference diagonals \pm 24 , excess sum diagonals $-22,-16,14,20$. For odd $x$ from -13 to $13, y$-values are $-9,13,-7,-1,5,9,-5,-11,11,3,-3,1,-13,7$; additional square $(-2,-2)$. All squares of row and column 14 of $Q_{29}$ are covered, so a copy of $Q_{28}$ is dominated. 
$i\left(Q_{29}\right), i\left(Q_{30}\right) \in\{15,16\}$ : 0-cover of size 16 for $Q_{29}$ with $e=4, f=2, u=3$, excess difference diagonal 18, excess sum diagonals $-24,-20,10,14,20$. For even $x$ from -14 to $14, y$-values are $-10,-4,6,12,-14,-8,4,-2,14,-12,8,2,10,0,-6$; additional square $(-9,9)$. All squares of column -15 and row 15 of $Q_{31}$ are covered, so a copy of $Q_{30}$ is dominated.

$i\left(Q_{34}\right) \in\{17,18\}$ : 0-cover of size 18 for $Q_{33}$ with $e=f=4, u=3$, excess difference diagonals $-24,24,28$, excess sum diagonals $-32,10,24$. For even $x$ from -16 to 16 , $y$-values are $-16,-6,4,10,16,-10,2,-4,-12,14,6,0,12,-14,-8,-2,8$; additional square $(-13,15)$. All squares of row and column 17 of $Q_{35}$ are covered, so a copy of $Q_{34}$ is dominated.

$i\left(Q_{35}\right), \gamma\left(Q_{35}\right), i\left(Q_{36}\right), \gamma\left(Q_{36}\right) \in\{18,19\}$ : 1-cover of size 19 for $Q_{35}$ with $e=6, f=3$, $u=3$, excess sum diagonals $-32,-22,-8,12,22,32$. For odd $x$ from -17 to $17, y$-values are $3,-17,-9,13,7,-11,1,9,-7,11,-13,-5,15,-15,-1,5,17,-3$; additional square $(2,2)$. All squares of row and column -18 of $Q_{37}$ are covered, so a copy of $Q_{36}$ is dominated.

$i\left(Q_{37}\right), i\left(Q_{38}\right) \in\{19,20\}$ : 0-cover of size 20 for $Q_{37}$ with $e=5, f=3, u=4$, excess difference diagonal 12, excess sum diagonals $-28,-26,8,26,30$. For even $x$ from -18 to $18, y$-values are $-8,6,-14,14,8,-10,2,-18,-12,4,-2,18,12,0,-16,-6,16$, $10,-4$; additional square $(-1,11)$. All squares of $Q_{39}$ except $(11,-19)$ are covered, so a copy of $Q_{38}$ is dominated.

$i\left(Q_{39}\right), i\left(Q_{40}\right) \in\{20,21\}: 1$-cover of size 21 for $Q_{39}$ with $e=4, f=3, u=5$, excess difference diagonals \pm 30 , excess sum diagonals $-20,8,12,20$. For odd $x$ from -19 to 19 , $y$-values are $9,-9,-3,17,5,15,-13,1,-19,19,-1,-17,7,-5,13,3,-15,11,-7$, -11 ; additional square $(10,10)$. All squares of row and column 20 of $Q_{41}$ are covered, so a copy of $Q_{40}$ is dominated.

$i\left(Q_{41}\right), i\left(Q_{42}\right) \in\{21,22\}$ : 0-cover of size 22 for $Q_{41}$ with $e=5, f=3, u=5$, excess difference diagonal 34, excess sum diagonals $-30,-28,8,20,30$. For even $x$ from -20 to $20, y$-values are $10,4,-14,-4,-16,-12,18,-20,0,16,8,2,-6,20,14,-8,-18$, $6,-10,12,-2$; additional square $(-17,17)$. All squares of $Q_{43}$ except $(13,-21)$ are covered, so a copy of $Q_{42}$ is dominated.

$i\left(Q_{43}\right), \gamma\left(Q_{43}\right), i\left(Q_{44}\right), \gamma\left(Q_{44}\right) \in\{22,23\}$ : 1-cover of size 23 for $Q_{43}$ with $e=6, f=3$, $u=5$, excess sum diagonals $-36,-30,8,12,20,30$. For odd $x$ from -21 to $21, y$-values are $-9,13,3,-21-5,-15,15,21,5,-19,-3,7,19,-7,-17,11,1,17,-13,9,-1$, -11 ; additional square $(2,2)$. All squares of row and column 22 of $Q_{45}$ are covered, so a copy of $Q_{44}$ is dominated.

$i\left(Q_{46}\right) \in\{23,24\}$ (and $i\left(Q_{45}\right)=\gamma\left(Q_{45}\right)=23$ ): begin with a 0-cover of size 23 for $Q_{45}$ from [2] with $e=5, f=3, u=6$, excess sum diagonals $-34,-12,8,38$. For even $x$ from -22 to $22, y$-values are $12,-10,0,10,-12,-22,20,14,-6,4,-20,-4,6,-18,-2$, $22,16,-14,8,2,-16,18,-8$. By Proposition 8 (B), adding the square $(15,-23)$ gives $i\left(Q_{46}\right) \in\{23,24\}$.

$i\left(Q_{47}\right), \gamma\left(Q_{47}\right), i\left(Q_{48}\right), \gamma\left(Q_{48}\right) \in\{24,25\}$ : 1-cover of size 25 for $Q_{47}$ with $e=4, f=3$, $u=7$, excess difference diagonals 10,22 , excess sum diagonals $\pm 28, \pm 12$. For odd $x$ from -23 to $23, y$-values are $-3,15,-11,7,-13,-21,-1,-9,21,17,-19,5,-5,19,1,11$, 
$-23,23,-15,13,-7,-17,9,3$; additional square $(-16,16)$. All squares of column -24 and row 24 are covered, so a copy of $Q_{48}$ is dominated.

$i\left(Q_{49}\right), i\left(Q_{50}\right) \in\{25,26\}$ : 0-cover of size 26 for $Q_{49}$ with $e=6, f=4, u=6$, excess difference diagonal 14, excess sum diagonals $-36,-30,10,36,40$. For even $x$ from -24 to $24, y$-values are $12,6,-12,-2,-20,-10,-18,14,24,0,-24,8,2,22,-6,4,20,-14$, $-4,-22,-16,18,-8,10,16$; additional square $(3,17)$. All squares of row and column 25 of $Q_{51}$ are covered, so a copy of $Q_{50}$ is dominated.

$i\left(Q_{51}\right), \gamma\left(Q_{51}\right), i\left(Q_{52}\right), \gamma\left(Q_{52}\right) \in\{26,27\}: 1$-cover of size 27 for $Q_{51}$ with $e=5, f=4$, $u=7$, excess difference diagonals -12 , 20, excess sum diagonals $-40,10,14,40$. For odd $x$ from -25 to $25, y$-values are $-7,-13,9,-5,21,-25,7,-9,25,15,-23,23,3,-3$, $-19,5,-1,-17,17,1,-15,-21,13,19,-11,11$ additional square $(8,16)$. All squares of $Q_{53}$ except $(16,-26)$ are covered, so a copy of $Q_{52}$ is dominated.

$i\left(Q_{53}\right), i\left(Q_{54}\right) \in\{27,28\}$ : 0-cover of size 28 for $Q_{53}$ with $e=7, f=5, u=6$, excess difference diagonal 16, excess sum diagonals $-46,-38,12,42,44$. For even $x$ from -26 to $26, y$-values are $-12,-6,16,-26,4,14,20,-10,-24,-18,6,0,24,-8,10,-22$, $2,-4,-20,18,12,26,-16,-2,22,-14,8$; additional square $(-1,15)$. All squares of column -27 and row 27 of $Q_{55}$ are covered, so a copy of $Q_{54}$ is dominated.

$i\left(Q_{55}\right), \gamma\left(Q_{55}\right), i\left(Q_{56}\right), \gamma\left(Q_{56}\right) \in\{28,29\}$ : 1-cover of size 29 for $Q_{55}$ with $e=7, f=6$, $u=6$, excess difference diagonals $-48,54$, excess sum diagonals $-40,-14,18,38$. For odd $x$ from -27 to $27, y$-values are $27,9,3,17,11,-19,-13,-27,1,-15,-25,-9,-3$, $21,15,7,-7,25,19,-23,5,23,-5,-11,-17,13,-1,-21$; additional square $(-2,4)$. All squares of row and column -28 of $Q_{57}$ are covered, so a copy of $Q_{56}$ is dominated.

$i\left(Q_{57}\right)=29, i\left(Q_{58}\right) \in\{29,30\}$ (and $\left.\gamma\left(Q_{57}\right)=29\right)$ : 0-cover of size 29 for $Q_{57}$ with $e=7, f=5, u=7$, excess sum diagonals $-42,-40,28,54$. For even $x$ from -28 to 28 , $y$-coordinates are $14,8,-10,-16,-20,-8,-26,24,-18,2,10,20,4,28,22,-6,0$, $-28,-14,-2,16,-24,18,-12,-22,-4,6,12,26$. By Proposition 8 (B), adding the square $(17,-29)$ shows $i\left(Q_{58}\right) \in\{29,30\}$.

$i\left(Q_{59}\right), \gamma\left(Q_{59}\right), i\left(Q_{60}\right), \gamma\left(Q_{60}\right) \in\{30,31\}: 1$-cover of size 31 for $Q_{59}$ with $e=8, f=5$, $u=7$, excess sum diagonals $-48,-42,-12,20,42,44$. For odd $x$ from -29 to 29, $y$-values are $-19,1,-17,17,-1,-15,27,21,11,-27,5,-5,-13,29,-29,-9,19,3$, $-7,-23,23,7,-25,13,25,-3,-21,-11,15,9$; additional square $(2,2)$. All squares of row and column -30 of $Q_{61}$ are covered, so a copy of $Q_{60}$ is dominated.

$i\left(Q_{62}\right) \in\{31,32\}$ : 0-cover of size 32 for $Q_{61}$ with $e=f=u=7$, excess difference diagonals $-44,-16,60$, excess sum diagonals $-44,16,58$. For even $x$ from -30 to 30 , $y$-values are $30,-6,-16,18,12,-24,20,-2,-8,-26,16,4,24,-22,6,-30,-10$, $-18,10,26,2,-4,8,-28,-20,22,-12,14,0,-14,28$; additional square $(15,15)$. All squares of $Q_{63}$ except $(-31,-15)$ are covered, so a copy of $Q_{62}$ is dominated.

$i\left(Q_{63}\right), \gamma\left(Q_{63}\right), i\left(Q_{64}\right), \gamma\left(Q_{64}\right) \in\{32,33\}:$ 1-cover of size 33 for $Q_{63}$ with $e=8, f=5$, $u=8$, excess sum diagonals $-46,-36,-16,12,44,46$. For odd $x$ from -31 to 31 , $y$-values are $-7,3,-19,23,13,-9,-17,27,-27,-21,29,1,7,11,25,-15,-5,9$, $-23,-29,-1,31,-11,19,-31,-25,17,21,5,-13,-3,15$; additional square $(2,2)$. All squares of row and column 32 are covered, so a copy of $Q_{64}$ is dominated.

$i\left(Q_{65}\right), i\left(Q_{66}\right) \in\{33,34\}$ : 0-cover of size 34 for $Q_{65}$ with $e=8, f=6, u=8$, excess 
difference diagonal 52 , excess sum diagonals $-48,-42,-14,48,58$. For even $x$ from -32 to $32, y$-values are $-4,18,-16,-6,0,-18,20,26,-32,-28,2,22,-20,30,-10,8$, $-2,-8,6,14,-24,-30,28,-22,-12,10,16,-26,24,32,4,-14,12$; additional square $(-25,27)$. All squares of $Q_{67}$ are covered except $(33,-19)$, so a copy of $Q_{66}$ is dominated.

$i\left(Q_{67}\right), \gamma\left(Q_{67}\right), i\left(Q_{68}\right), \gamma\left(Q_{68}\right) \in\{34,35\}$ : 1-cover of size 35 for $Q_{67}$ with $e=7, f=6$, $u=9$, excess difference diagonals $-20,24$, excess sum diagonals $-52,-14,38,52$. For odd $x$ from -33 to $33, y$-values are $5,-1,-7,-21,-27,11,25,-25,33,27,13,-9$, $-31,17,3,9,-5,-11,-17,-3,-23,-33,29,23,-19,31,19,-29,-15,7,1,15,21$, -13 ; additional square $(10,14)$. All squares of row and column -34 of $Q_{69}$ are covered, so a copy of $Q_{68}$ is dominated.

$i\left(Q_{69}\right)=35$ (and $\left.\gamma\left(Q_{69}\right)=35\right)$ : 0-cover with $e=8, f=6, u=9$, excess sum diagonals $\pm 38, \pm 56$. For even $x$ from -34 to $34, y$-values are $-22,-8,18,8,-10,-24,30,-18$, $22,28,-30,-16,10,6,26,-28,4,-6,-12,2,34,-32,0,-20,24,20,-34,12,-26$, $32,-2,16,-14,-4,14$.

$i\left(Q_{70}\right) \in\{35,36\}$ : 0-cover of size 36 for $Q_{69}$ with $e=f=7, u=9$, excess difference diagonals $-52,16,40$, excess sum diagonals $-44,16,62$. For even $x$ from -34 to $34, y$ values are $-12,6,-14,-2,4,-26,28,22,16,30,26,-22,-28,-34,2,14,10,-8,-20$, $0,-6,8,20,-18,-24,-30,32,-32,24,18,12,34,-4,-10,-16$; additional square $(15,19)$. All squares of $Q_{71}$ except $( \pm 19,35)$ are covered, so a copy of $Q_{70}$ is dominated.

$\gamma\left(Q_{71}\right)=36$ : 1-cover with $e=6, f=5, u=11$, excess difference diagonal 0, excess sum diagonals $0, \pm 2$. For odd $x$ from -35 to $35, y$-values are $-19,-13,-3,15,25,-25$, $17,35,-11,19,33,-29,21,-17,-31,5,-5,1,7,-7,-1,31,-27,11,-35,27,-15$, $-21,9,-33,29,23,-23,3,13,-9$.

$i\left(Q_{71}\right), i\left(Q_{72}\right) \in\{36,37\}$ : 1-cover of size 37 for $Q_{71}$ with $e=7, f=6, u=10$, excess difference diagonals $-16,28$, excess sum diagonals $-56,14,42,56$. For odd $x$ from -35 to $35, y$-values are $-9,9,-21,-27,11,21,-5,33,-29,17,35,-19,-25,19,15,-35$, $-3,3,31,-13,-7,-1,5,-31,27,-23,25,-15,-33,29,23,1,7,13,-17,-11$; additional square $(22,34)$. All squares of $Q_{73}$ but $(-36,22)$ are covered, so a copy of $Q_{72}$ is dominated.

$i\left(Q_{73}\right)=37$ (and $\left.\gamma\left(Q_{73}\right)=37\right)$ : 0-cover with $e=8, f=6, u=10$, excess sum diagonals $\pm 18, \pm 60$. For even $x$ from -36 to $36, y$-values are $-24,14,24,-6,0,26,12$, $-30,-20,22,-28,-34,32,-8,-2,4,10,30,-32,-26,-10,-4,2,8,28,18,36,-22$, $-36,-14,20,34,-16,6,16,-18,-12$.

$i\left(Q_{74}\right) \in\{37,38\}$ : 0-cover of size 38 for $Q_{73}$ with $e=f=7, u=10$, excess difference diagonals $-36,16,24$, excess sum diagonals $-48,16,70$. For even $x$ from -36 to 36 , $y$-values are $-14,8,22,0,-20,20,-30,-12,-22,32,18,-32,12,6,30,-16,-34$, $-6,26,14,4,24,-4,2,-24,-28,-10,-36,34,28,-26,-8,-18,16,10,36,-2$; additional square $(17,21)$. All squares of $Q_{75}$ except $( \pm 21,-37)$ are covered, so a copy of $Q_{74}$ is dominated.

$i\left(Q_{75}\right), \gamma\left(Q_{75}\right), i\left(Q_{76}\right), \gamma\left(Q_{76}\right) \in\{38,39\}$ : 1-cover of size 39 for $Q_{75}$ with $e=10$, $f=7, u=9$, excess sum diagonals $-68,-54,-16,36,54,60$. For odd $x$ from -37 to $37, y$-values are $-31,-15,-5,13,27,-19,-29,25,15,33,23,3,-21,21,1,-23,-37$, $-13,-3,-27,5,-1,31,-9,-33,17,-25,29,35,-35,37,11,7,-7,19,9,-17,-11$; 
additional square $(6,6)$. All squares of row and column -38 of $Q_{77}$ are covered, so a copy of $Q_{76}$ is dominated.

$i\left(Q_{77}\right)=39$ (and $\left.\gamma\left(Q_{77}\right)=39\right)$ : 0-cover with $e=9, f=7, u=10$, excess sum diagonals $-62,-48,44,66$. For even $x$ from -38 to $38, y$-values are $-24,-18,-4,2,24$, $14,-22,-26,36,30,-28,22,32,-30,-16,4,-6,12,6,-34,-2,-12,28,34,16,0$, $-36,-14,-20,-38,-32,26,18,38,8,-10,20,10,-8$.

$i\left(Q_{78}\right) \in\{39,40\}$ : 0-cover of size 40 for $Q_{77}$ with $e=f=8, u=10$, excess difference diagonals $-38,18,38$, excess sum diagonals $-68,18,74$. For even $x$ from -38 to 38 , $y$-values are $-30,-20,-10,-4,22,-24,30,24,10,18,26,-16,-34,28,4,-32,-14$, $32,8,-28,-12,-2,12,6,0,-26,-38,36,-22,-36,34,20,14,-8,-18,16,2,38$, -6 ; additional square $(3,21)$. All squares of $Q_{79}$ except $( \pm 21,39)$ are covered, so a copy of $Q_{78}$ is dominated.

$i\left(Q_{79}\right), \gamma\left(Q_{79}\right), i\left(Q_{80}\right), \gamma\left(Q_{80}\right) \in\{40,41\}$ : 1-cover of size 41 for $Q_{79}$ with $e=10$, $f=7, u=10$, excess sum diagonals $-64,-58,-16,40,44,58$. For odd $x$ from -39 to $39, y$-values are $-25,-21,-15,-1,-23,31,25,-13,33,15,-27,23,29,-29,7$, $39,-19,-7,3,9,-17,-11,-35,35,13,1,37,-37,-31,21,-39,-33,19,-9,-3$, $27,5,11,17,-5$; additional square $(2,2)$. All squares of row and column -40 of $Q_{81}$ are covered, so a copy of $Q_{80}$ is dominated.

$i\left(Q_{81}\right)=41$ and $i\left(Q_{82}\right) \in\{41,42\}$ (and $\left.\gamma\left(Q_{81}\right)=41\right)$ : 0-cover with $e=9, f=7$, $u=11$, excess sum diagonals $-62,-36,28,70$. For even $x$ from -40 to $40, y$-values are $-22,24,-10,8,-26,28,6,20,-12,32,-30,-24,-38,36,26,-28,-4,2,12,-32$, $-8,-2,34,-36,22,-6,0,14,38,16,-18,-40,-34,-20,30,40,18,-16,10,4,-14$. By (B) of Proposition 8, adding the square $(25,-41)$ shows $i\left(Q_{82}\right) \in\{41,42\}$.

$i\left(Q_{83}\right), \gamma\left(Q_{83}\right), i\left(Q_{84}\right), \gamma\left(Q_{84}\right) \in\{42,43\}, i\left(Q_{85}\right)=43$ (and $\left.\gamma\left(Q_{85}\right)=43\right)$ : 1-cover of size 43 for $Q_{85}$ with $e=10, f=9, u=11, a=6$, excess sum diagonals $-44,56$. For odd $x$ from -41 to $41, y$-values are $-21,25,11,-3,-9,-15,27,33,-33,-27,15,-25,-37$, $29,39,-5,31,3,9,-31,-11,-1,5,-13,-29,-39,23,41,1,21,37,-19,-41,-35$, $35,13,19,-23,-17,-7,7,17$, and additional square $(6,6)$. As the set has no squares on the edges of $Q_{85}$, it is also a dominating set of $Q_{83}$ and $Q_{84}$.

$i\left(Q_{86}\right) \in\{43,44\}$ : 0-cover of size 44 for $Q_{85}$ with $e=10, f=8, u=11$, excess difference diagonal 26, excess sum diagonals $-64,-62,18,64,68$. For even $x$ from -42 to $42, y$-values are $18,-8,-18,8,-28,16,-6,-16,-38,40,14,-12,-42,36,42,-40$, $4,10,-4,-36,38,-14,12,2,34,-10,0,6,-34,32,-22,-32,22,-20,30,24,-30$, $-24,-2,28,-26,20,26$; additional square $(-1,25)$. All squares of $Q_{87}$ except $(25,-43)$ are covered, so a copy of $Q_{86}$ is dominated.

$i\left(Q_{87}\right), \gamma\left(Q_{87}\right), i\left(Q_{88}\right), \gamma\left(Q_{88}\right) \in\{44,45\}:$ 1-cover of size 45 for $Q_{87}$ with $e=9, f=8$, $u=12$, excess difference diagonals \pm 40 , excess sum diagonals $-68,18,22,68$. For odd $x$ from -43 to $43, y$-values are $-9,-19,15,9,-21,17,-1,33,-37,-43,43,21,-25$, $41,23,-35,5,31,-33,3,-7,-13,13,-39,-3,11,19,-5,39,-23,-29,37,-41,25$, $-15,-31,35,29,-17,1,7,-27,27,-11$; additional square $(20,20)$. All squares of row and column 44 of $Q_{89}$ are covered, so a copy of $Q_{88}$ is dominated.

$i\left(Q_{89}\right)=\gamma\left(Q_{89}\right)=45$ and $i\left(Q_{90}\right) \in\{45,46\}: 0$-cover with $e=11, f=9, u=11$, excess sum diagonals $-80,-70,66,84$. For even $x$ from -44 to $44, y$-values are -36 , 
$-28,-22,-16,-2,-24,22,0,38,20,-18,36,42,24,34,-32,4,-40,12,6,-8,-14$, $-34,40,30,-10,8,2,-30,-6,-42,-20,10,-44,-38,28,-26,16,26,32,14,-12$, $44,-4,18$. By (B) of Proposition 8, adding the square $(-25,45)$ shows $i\left(Q_{90}\right) \in\{45,46\}$.

$\gamma\left(Q_{91}\right)=46$ : 1-cover with $e=9, f=8, u=13$, excess difference diagonal 0, excess sum diagonals $-70,8,62$. For odd $x$ from -45 to $45, y$-values are $13,-27,-15,27,9$, $15,-35,-9,41,35,17,-21,-39,-45,-31,19,-1,-41,45,-3,33,5,-5,-37,3$, $-7,7,1,29,43,-19,-33,-43,31,-23,39,21,-13,37,11,25,-29,23,-17,-11$, -25 .

$i\left(Q_{91}\right), i\left(Q_{92}\right) \in\{46,47\}:$ 1-cover of size 47 for $Q_{91}$ with $e=11, f=10, u=11$, excess difference diagonals \pm 88 , excess sum diagonals $-68,-22,38,68$. For odd $x$ from -45 to $45, y$-values are $43,15,-7,-17,17,27,-19,-1,13,-33,-39,-45,45,-21$, $21,1,-5,39,-35,-15,41,9,19,-11,7,-41,-13,5,37,-37,-27,35,25,-9,33$, $-29,11,31,-31,-25,-3,3,29,23,-23,-43$; additional square (8,8). All squares of row and column -46 of $Q_{93}$ are covered, so a copy of $Q_{92}$ is dominated.

$i\left(Q_{93}\right)=\gamma\left(Q_{93}\right)=47$ : 0-cover with $e=11, f=9, u=12$, excess sum diagonals -82 , $-60,52,90$. For even $x$ from -46 to $46, y$-values are $-36,-22,-8,30,-24,6,-26$, $-14,28,38,20,-30,40,-38,32,22,-28,42,2,-4,10,16,-32,0,-18,-42,-10,34$, $-2,4,44,-34,-44,18,-20,-46,-40,24,36,-6,12,26,-16,14,8,46,-12$.

$i\left(Q_{94}\right) \in\{47,48\}$ : 0-cover of size 48 for $Q_{93}$ with $e=11, f=9, u=12$, excess difference diagonal 74 , excess sum diagonals $-70,-48,-36,70,88$. For even $x$ from -46 to $46, y$-values are $-24,6,-16,-6,20,10,32,-30,40,34,28,-26,-32,-46,-18,4$, $-34,30,2,22,-28,-12,14,-4,-44,12,44,-8,-2,-42,-36,-22,24,38,16,-38$, $36,42,-40,-10,0,18,8,26,46,-14,-20$; additional square $(-35,39)$. All squares of column -47 and row 47 of $Q_{95}$ are covered, so a copy of $Q_{94}$ is dominated.

$i\left(Q_{95}\right), \gamma\left(Q_{95}\right), i\left(Q_{96}\right), \gamma\left(Q_{96}\right) \in\{48,49\}$ : 1-cover of size 49 for $Q_{95}$ with $e=12$, $f=9, u=12$, excess sum diagonals $-70,-64,-40,20,70,92$. For odd $x$ from -47 to $47, y$-values are $25,19,13,-5,29,-33,-29,27,-35,-21,-13,-37,-31,31,-39,23$, $17,35,-27,9,3,-11,41,21,-9,-45,-17,5,37,-7,-47,1,33,43,-23,-41,45,39$, $-43,-1,-19,7,-3,15,-15,-25,47,11$; additional square $(4,4)$. All squares of row and column 48 of $Q_{97}$ are covered, so a copy of $Q_{96}$ is dominated.

$i\left(Q_{97}\right)=\gamma\left(Q_{97}\right)=49$ and $i\left(Q_{98}\right) \in\{49,50\}$ : 0-cover with $e=12, f=10, u=12$, excess sum diagonals $-90,-78,72,96$. For even $x$ from -48 to $48, y$-values are -42 , $-32,-24,-18,0,-26,-20,-2,36,-22,24,46,32,38,44,18,28,-30,6,12,40$, $-8,-44,-16,-10,-34,14,8,2,-12,-6,42,-36,-46,-28,-38,-48,22,-40,34$, 20, 10, 4, 30, -4, $-14,16,26,48$. By (B) of Proposition 8 , adding the square $(-27,49)$ shows $i\left(Q_{98}\right) \in\{49,50\}$.

$\gamma\left(Q_{99}\right), \gamma\left(Q_{100}\right) \in\{50,51\}$ and $\gamma\left(Q_{101}\right)=51$ : 1-cover of size 51 for $Q_{101}$ with $e=11$, $f=10, u=14, a=29$, excess sum diagonals 8, 50. For odd $x$ from -49 to $49, y$-values are $-27,31,17,-29,5,-13,-31,39,-23,27,37,-33,45,-21,21,-45,33,1,-35$, $43,11,-3,3,-15,-39,13,41,-11,-1,-41,7,47,-5,35,49,19,-47,25,-19,-49$, $-43,23,-7,-25,9,-17,29,-9,29,15$; additional square $(29,-37)$. As the set has no squares on the edges of $Q_{101}$, it is also a dominating set for $Q_{99}$ and $Q_{100}$.

$i\left(Q_{99}\right), i\left(Q_{100}\right) \in\{50,51\}: 1$-cover of size 51 for $Q_{99}$ with $e=12, f=9, u=13$, 
excess sum diagonals $-74,-52,-36,20,74,84$. For odd $x$ from -49 to $49, y$-values are $27,17,-29,29,-1,-27,31,1,-37,-21,19,33,-33,21,35,-35,-19,37,-49,11$, $-41,-5,13,-13,-45,-39,9,-9,-15,41,-7,7,43,-43,23,45,3,-23,47,-47,39$, $5,49,25,15,-31,-25,-11,-17,-3$; additional square $(8,8)$. All squares of row and column 50 of $Q_{101}$ are covered, so a copy of $Q_{100}$ is dominated.

$i\left(Q_{101}\right), i\left(Q_{102}\right) \in\{51,52\}$ : 0-cover of size 52 for $Q_{101}$ with $e=f=10, u=14$, excess difference diagonals $-66,22,46$, excess sum diagonals $-62,22,80$. For even $x$ from -50 to $50, y$-values are $-26,-12,-18,16,-20,0,-6,36,-34,44,14,20,30,-8,-50,48$, $28,-36,50,-44,42,-2,4,10,-46,18,-16,-10,-4,6,-30,-48,8,-40,26,-32$, $-14,-42,46,40,34,24,-38,12,22,-24,38,32,-22,-28,2$; additional square $(19,21)$. All squares of row and column 51 of $Q_{103}$ are covered, so a copy of $Q_{102}$ is dominated.

$i\left(Q_{103}\right), \gamma\left(Q_{103}\right), i\left(Q_{104}\right), \gamma\left(Q_{104}\right) \in\{52,53\}, i\left(Q_{105}\right)=\gamma\left(Q_{105}\right)=53$ : 1-cover of size 53 for $Q_{105}$ with $e=12, f=11, u=14, a=4$, excess sum diagonals $-52,60$. For odd $x$ from -51 to $51, y$-values are $-27,31,-7,-29,13,7,-31,35,29,-13,-21,39,-39$, $51,37,-37,25,-25,-47,9,3,43,-43,-11,15,-19,-1,-41,-17,-3,11,47,41,1$, $49,23,17,-49,-35,33,-51,-45,45,-33,-15,-9,-23,19,5,27,21,-5$; additional square $(4,4)$. As the set has no squares on the edges of $Q_{105}$, it is also a dominating set for $Q_{103}$ and $Q_{104}$.

$i\left(Q_{106}\right) \in\{53,54\}$ : 0 -cover of size 54 for $Q_{105}$ with $e=12, f=10, u=14$, excess difference diagonal 26, excess sum diagonals $-84,-62,22,80,92$. For even $x$ from -52 to $52, y$-values are $-32,30,16,-14,32,-34,0,34,-36,-28,36,-38,-24,26,-40$, $-26,40,4,28,-42,44,8,-10,42,6,-8,-44,38,18,-12,10,-4,2,-50,-6,46$, $-48,-30,-16,50,-52,-46,48,22,-20,14,52,-18,24,-2,12,-22,20$; additional square $(11,37)$. All squares of row and column 53 of $Q_{107}$ are covered, so a copy of $Q_{106}$ is dominated.

$i\left(Q_{107}\right), \gamma\left(Q_{107}\right), i\left(Q_{108}\right), \gamma\left(Q_{108}\right) \in\{54,55\}$ : 1-cover of size 55 for $Q_{107}$ with $e=10$, $f=9, u=16$, excess difference diagonals 22,42 , excess sum diagonals $-36,-28,20,48$. For odd $x$ from -53 to $53, y$-values are $23,33,-33,21,35,5,-5,-35,-25,37,-45$, $-39,27,-31,-41,29,-15,-9,-37,-1,47,11,31,-43,13,41,-7,-13,45,7,-11$, $-51,9,3,-49,49,39,-27,51,25,-17,53,-53,-47,43,-19,15,1,-29,17,-21$, $-3,19,-23$; additional square $(-30,34)$. All squares of column -54 and row 54 of $Q_{109}$ are covered, so a copy of $Q_{108}$ is dominated.

$i\left(Q_{109}\right)=\gamma\left(Q_{109}\right)=55$ : 0-cover with $e=13, f=11, u=14$, excess sum diagonals $-94,-86,72,108$. For even $x$ from -54 to $54, y$-values are $-40,-34,-28,34,-4,2$, $36,-30,-36,26,40,-26,-32,-38,24,46,32,38,48,-22,-6,0,-44,8,14,-12,22$, $-46,-10,42,10,4,-14,-8,44,-50,52,-42,-52,50,-24,-54,-48,18,-20,6,16$, $-2,-16,28,20,30,12,-18,54$.

$i\left(Q_{110}\right) \in\{55,56\}$ : 0 -cover of size 56 for $Q_{109}$ with $e=f=11, u=15$, excess difference diagonals $-60,36,44$, excess sum diagonals $-84,24,86$. For even $x$ from -54 to $54, y$-values are $-16,-10,-34,18,32,6,16,-14,-4,-30,48,22,-48,-54,4$, $46,-52,54,-28,20,30,0,52,-50,-44,42,-2,8,-18,-12,-6,12,2,-46,10,34$, $40,-40,-24,-42,28,-26,-20,-38,44,50,36,26,-36,38,24,14,-32,-22,-8$; additional square $(3,23)$. All squares of row and column 55 of $Q_{111}$ are covered, so a copy 
of $Q_{110}$ is dominated.

$i\left(Q_{111}\right), \gamma\left(Q_{111}\right), i\left(Q_{112}\right), \gamma\left(Q_{112}\right) \in\{56,57\}$ : 1-cover of size 57 for $Q_{111}$ with $e=13$, $f=12, u=14$, excess difference diagonals $-100,108$, excess sum diagonals $-84,-26$, 50,84 . For odd $x$ from -55 to $55, y$-values are $27,55,-5,-19,11,33,19,-23,31,37$, $15,-27,-49,-55,39,-47,-53,3,-21,25,1,-13,9,-43,-15,-1,-41,53,13,-51$, $-37,-17,47,7,-7,49,5,41,23,-39,51,21,43,-35,17,45,-33,29,-9,-3,-29$, $-11,35,-31,-25,-45$; additional square $(8,16)$. All squares of row and column -56 of $Q_{113}$ are covered, so a copy of $Q_{112}$ is dominated.

$\gamma\left(Q_{113}\right)=57$ : 0 -cover with $e=f=11, u=16$, excess difference diagonals \pm 88 , excess sum diagonals \pm 20 . This set is symmetric by a half-turn about board center, so we only give the $y$-values for even $x$ from 0 to $56: 0,-48,-12,44,-4,52,8,6,-42,-2$, $22,56,50,-20,-46,36,-54,24,18,-40,-30,28,-10,-16,-34,-38,-14,32,26$.

$i\left(Q_{113}\right), i\left(Q_{114}\right) \in\{57,58\}: 0$-cover of size 58 for $Q_{113}$ with $e=13, f=11, u=15$, excess difference diagonal 90, excess sum diagonals $-92,-86,36,76,86$. For even $x$ from -56 to $56, y$-values are $-36,-32,34,-24,14,-12,6,16,30,36,-26,-20,50,-40$, $-54,20,54,-56,22,-48,-34,52,-4,44,8,18,-42,-6,4,-22,-16,-10,0,-52$, $24,2,46,56,-50,-44,-18,32,26,48,-46,12,-14,38,42,28,-30,40,-38,-8,-2$, $-28,10$; additional square $(-35,55)$. All squares of column -57 and row 57 of $Q_{115}$ are covered, so a copy of $Q_{114}$ is dominated.

$\gamma\left(Q_{115}\right)=58$ : 1 -cover with $e=11, f=10, u=17$, excess difference diagonal 0 , excess sum diagonals $0, \pm 54$. For odd $x$ from -57 to $57, y$-values are $-31,-25,-19,15,29$, $-9,13,43,-35,11,-17,55,-15,31,53,-57,-27,51,-43,27,-1,-53,57,-3,-51$, $47,-5,9,3,-7,-47,5,49,-11,7,1,-55,-41,33,-33,45,-21,21,-37,41,-49$, $19,39,25,-45,17,-29,37,-13,-39,35,-23,23$.

$i\left(Q_{115}\right), i\left(Q_{116}\right) \in\{58,59\}: 1$-cover of size 59 for $Q_{115}$ with $e=14, f=11, u=15$, excess sum diagonals $-104,-86,-24,56,72,86$. For odd $x$ from -57 to $57, y$-values are $-47,-31,-25,-19,-33,-27,43,1,-5,25,31,-23,47,53,19,33,-37,-43,51,57$, $35,41,-11,-39,-3,15,9,-17,-53,-9,-41,-21,5,-13,-45,7,55,-1,37,29$, $49,-55,-49,13,-57,-51,27,-35,-29,45,39,21,11,-15,3,-7,23,17$; additional square $(0,0)$. All squares of row and column -58 of $Q_{117}$ are covered, so a copy of $Q_{116}$ is dominated.

$\gamma\left(Q_{117}\right)=59$ : 0 -cover with $e=13, f=11, u=16$, excess sum diagonals $-80,-78$, 58,100 . For even $x$ from -58 to $58, y$-values are $-16,-22,-24,-6,-12,18,4,26,44$, $50,-32,42,28,-18,-56,-52,56,-58,-44,54,36,-30,-2,12,48,-46,14,-4,6$, $-20,-14,-8,10,-42,2,8,-40,-54,40,46,-36,34,24,-50,32,22,52,-38,-48$, $-26,58,38,0,30,20,-10,-28,-34,16$.

$i\left(Q_{117}\right), i\left(Q_{118}\right) \in\{59,60\}$ : 0-cover of size 60 for $Q_{117}$ with $e=f=12, u=16$, excess difference diagonals $-102,26,92$, excess sum diagonals $-62,34,50$. For even $x$ from -58 to $58, y$-values are $-30,36,-22,-32,38,4,-10,-36,-20,0,-34,48,34,40,-38$, $20,54,-28,42,56,-46,-6,46,14,8,-52,-42,52,12,-18,-8,10,-16,-44,-4,6$, $58,-48,16,44,22,26,-54,-12,-26,-56,-50,-40,50,28,18,-58,30,-24,2,32$, $-14,24,-2$; additional square $(3,19)$. All squares of column -59 and row 59 of $Q_{119}$ are covered, so a copy of $Q_{118}$ is dominated. 
$i\left(Q_{119}\right), \gamma\left(Q_{119}\right), i\left(Q_{120}\right), \gamma\left(Q_{120}\right) \in\{60,61\}$ : 1-cover of size 61 for $Q_{119}$ with $e=14$, $f=11, u=16$, excess sum diagonals $-90,-84,-24,32,76,90$. For odd $x$ from -59 to $59, y$-values are $-31,35,21,-33,-15,-35,37,-37,45,3,25,43,-39,27,-27,-49$, $-43,47,33,-29,49,31,-9,9,-51,-11,-5,13,7,-45,-55,-19,-47,59,-17,5$, $-1,39,57,1,11,55,51,-57,41,19,-59,-53,53,-41,17,-25,-3,15,-23,-13$, $-7,-21,29,23$; additional square $(0,0)$. All squares of row and column -60 of $Q_{121}$ are covered, so a copy of $Q_{120}$ is dominated.

$\gamma\left(Q_{121}\right)=61$ : 0-cover with $e=13, f=11, u=17$, excess sum diagonals $-74,-64$, 40, 98. For even $x$ from -60 to $60, y$-values are $26,36,18,24,38,-40,-6,-32,2,40$, $30,-36,-28,28,-42,-56,-30,-44,-58,44,34,-48,0,6,-50,14,50,-14,-46$, $10,-4,52,-16,-52,8,48,-12,-2,4,22,54,16,42,56,-26,-20,58,-60,-54,60$, $46,20,-38,32,-18,-24,-34,-8,-22,12,-10$.

$\gamma\left(Q_{125}\right)=63$ : 0-cover with $e=13, f=11, u=18$, excess sum diagonals $-68,-40$, 18, 90. For even $x$ from -62 to $62, y$-values are $-32,-26,-20,22,-36,30,20,-6,0$, $42,24,-18,56,62,28,-30,60,-12,-44,-50,-46,-62,6,58,-52,-8,48,4,14$, $-4,52,-16,10,54,-2,-58,-10,8,2,-54,-60,-34,32,50,40,18,-56,-42,-24$, $-14,44,-22,36,-38,16,46,-48,38,-40,34,12,26,-28$.

$\gamma\left(Q_{129}\right)=\gamma\left(Q_{130}\right)=65$ : 0-cover of $Q_{129}$ with $e=14, f=12, u=18$, excess sum diagonals $-100,-26,0,126$. For even $x$ from -64 to $64, y$-values are $28,22,-16,14$, $-24,-2,-12,-22,0,54,24,46,-56,58,-64,-58,32,26,-60,-34,36,-62,56,62$, $-52,-8,10,4,18,12,-10,-50,-26,-20,-14,16,-6,8,2,38,-48,-42,-32,42$, $60,-54,-44,-38,48,34,40,50,20,30,52,-46,44,6,-4,-30,-40,-18,-28,64$, -36 . We may apply Proposition $6(\mathrm{~A})$ with $k=32$; since there are excess sum diagonals numbered -100 and -26 , this set dominates $Q_{130}$.

$\gamma\left(Q_{131}\right)=66$ : 1-cover with $e=14, f=13, u=18$, excess difference diagonal 0 , excess sum diagonals $-130,2,128$. Take the set on $Q_{129}$ just given, add 1 to each row and column number, and include the square $(-65,-65)$.

Below we summarize established values and bounds for $\gamma\left(Q_{n}\right)$ and $i\left(Q_{n}\right)$; values from [19] are in italic, those from [14] are underlined, those from this work are in bold, and older ones are in roman type.

$i\left(Q_{n}\right)=\gamma\left(Q_{n}\right)=(n-1) / 2$ for $n=3,11$.

$\gamma\left(Q_{n}\right)=\lceil n / 2\rceil$ for $n=1,2,4-7,9,10,12,13,17-19,21,23,25,27,29-31,33,37,39$, $41,45,49,53,57,61, \underline{65}, 69, \mathbf{7 1}, \underline{73}, 77, \underline{81}, \underline{85}, \mathbf{8 9}, \mathbf{9 1}, \mathbf{9 3}, \mathbf{9 7}, \mathbf{1 0 1}, \mathbf{1 0 5}, \mathbf{1 0 9}, \mathbf{1 1 3}, \mathbf{1 1 5}$, 117, 121, 125, 129-131.

$\gamma\left(Q_{n}\right)=\lceil n / 2\rceil+1$ for $n=8, \underline{14}, \underline{15}, \underline{16}$.

$\gamma\left(Q_{n}\right) \in\{\lceil n / 2\rceil,\lceil n / 2\rceil+1\}$ for $n=20,22,24-26, \mathbf{2 8}, 32,34, \mathbf{3 5}, \mathbf{3 6}, 38,40,42, \mathbf{4 3}, \mathbf{4 4}$, $46, \mathbf{4 7}, \mathbf{4 8}, 50, \mathbf{5 1}, \mathbf{5 2}, 54, \mathbf{5 5}, \mathbf{5 6}, 58, \mathbf{5 9}, \mathbf{6 0}, 62, \mathbf{6 3}, \mathbf{6 4}, \underline{66}, \mathbf{6 7}, \mathbf{6 8}, \mathbf{7 0}, \mathbf{7 2}, \underline{\mathbf{7 4}}, \mathbf{7 5}, \mathbf{7 6}$, $78,79,80, \underline{82}, 83,84, \underline{86}, 87,88,90,92,94-96,98-100,102-104,106-108,110-112$, $114,116,118-120,122,126,132$. 
$i\left(Q_{n}\right)=\lceil n / 2\rceil$ for $n=1,2,5,7,9,10,13,17,21,25,33,45, \mathbf{5 7}, 61, \mathbf{6 9}, \mathbf{7 3}, \mathbf{7 7}, \mathbf{8 1}, \mathbf{8 5}$, 89, 93, 97, 105, 109.

$i\left(Q_{n}\right)=\lceil n / 2\rceil+1$ for $n=4,6,8,12,14-16, \underline{18}$.

$i\left(Q_{n}\right) \in\{\lceil n / 2\rceil,\lceil n / 2\rceil+1\}$ for $n=19,20, \underline{22}, 23,24, \mathbf{2 6 - 3 0}, 31,32, \mathbf{3 4 - 4 4}, \mathbf{4 6 - 5 6}$, 58-60, 62-68, 70-72, 74-76, 78-80, 82-84, 86-88, 90-92, 94-96, 98-104, 106-108, 110-120.

\section{Concluding Remarks}

Much previous work has depended on finding dominating sets symmetric by a half-turn about board center. This is not always possible, even when the line sets have the desired symmetry. Specifically, the dominating sets given above to establish the values of $i\left(Q_{69}\right)$ and $i\left(Q_{73}\right)$ each have the property that their sets of occupied lines have $180^{\circ}$ symmetry, but an exhaustive search showed there are no minimum dominating sets with $180^{\circ}$ symmetry that occupy these line sets.

From (1) and the definitions of $\gamma$ and $i$, we have $(n-1) / 2 \leq \gamma\left(Q_{n}\right) \leq i\left(Q_{n}\right)$ for all $n$; our results lead us to add the following conjecture.

Conjecture For all $n, i\left(Q_{n}\right) \leq\lceil n / 2\rceil+1$.

It would be very interesting to know if equality occurs in (1) for any $n$ other than 3 and 11. It is only necessary to examine the members of the sequence $\left(n_{i}\right)$ defined in Theorem 1. The smallest open case is $n_{4}=143$; it is shown in [19] that if $\gamma\left(Q_{143}\right)=71$, then any minimum dominating set of $Q_{143}$ is an independent 0 -cover with $e=f=15$, $u=20$, and no excess diagonals.

\section{References}

[1] W. Ahrens, Mathematische Unterhaltungen und Spiele (B. G. Teubner, Leipzig-Berlin, 1910).

[2] A. P. Burger And C. M. Mynhardt, Symmetry and domination in queens graphs, Bull. Inst. Combin. Appl. 29(2000), 11-24.

[3] A. P. Burger and C. M. Mynhardt, Properties of dominating sets of the queen's graph $Q_{4 k+3}$, Utilitas Math. 57(2000), 237-253.

[4] A. P. Burger and C. M. Mynhardt, An upper bound for the minimum number of queens covering the $n \times n$ chessboard, submitted.

[5] A. P. Burger, C. M. Mynhardt, and E. J. Cockayne, Domination numbers for the queen's graph, Bull. Inst. Combin. Appl. 10(1994), 73-82. 
[6] A. P. Burger, E. J. Cockayne, and C. M. Mynhardt, Domination and irredundance in the queen's graph, Discrete Math. 163(1997), 47-66.

[7] E. J. Cockayne, Chessboard domination problems, Discrete Math. 86(1990), $13-20$.

[8] M. Eisenstein, C. Grinstead, B. Hahne, and D. Van Stone, The queen domination problem, in: Proceedings of the Twenty-third Southeastern International Conference on Combinatorics, Graph Theory, and Computing, Congr. Numer. 91(1992), 189-193.

[9] P. Gibbons And J. WebB, Some new results for the queens domination problem, Australas. J. Combin. 15(1997), 145-160.

[10] R. K. GuY, Unsolved Problems in Number Theory, 2nd Edition (SpringerVerlag, New York, 1994).

[11] S. M. Hedetniemi, S. T. Hedetniemi, and R. Reynolds, Combinatorial problems on chessboards: II, in: Domination in Graphs: Advanced Topics, T. W. Haynes, S. T. Hedetniemi, and P. J. Slater (Editors), 133-162 (Marcel Dekker, New York, 1998).

[12] H. Hitotumatu and K. Noshita, A technique for implementing backtrack algorithms and its application, Inform. Process. Lett. 8(1979), 174-175.

[13] C. F. DE JAEnisch, Applications de l'Analyse Mathématique au Jeu des Échecs, Appendix, pages $244 \mathrm{ff}$ (Petrograd, 1862).

[14] M. D. Kearse And P. B. Gibbons, Computational methods and new results for chessboard problems, Australas. J. Combin. 23(2001), 253-284.

[15] D. E. Knuth, Dancing links, in: Millennial Perspectives in Computer Science, J. Davies, B. Roscoe, and J. Woodcock (Editors), 187-214 (Palgrave, Houndmills, 2000).

[16] W. W. Rouse BalL, Mathematical Recreations $\&$ Essays, 3rd Edition (revision by H. S. M. Coxeter of the original 1892 edition), Chapter 6, Minimum pieces problem (Macmillan, London, 1939).

[17] W. D. Weakley, Domination in the queen's graph, in: Graph Theory, Combinatorics, and Algorithms, Vol. 2 (Kalamazoo, MI 1992), Y. Alavi and A. Schwenk (Editors), 1223-1232 (Wiley, New York, 1995).

[18] W. D. WEAKLEY, Upper bounds for domination numbers of the queen's graph, to appear in Discrete Math.

[19] W. D. WEAKLEY, A lower bound for domination numbers of the queen's graph, submitted. 


\section{Comment by the authors added August 25th 2003}

In the above paper, the theorem that at least $(n-1) / 2$ queens are required to dominate the $n$ by $n$ chessboard was attributed to P. H. Spencer, in [1]. A proof of this result appeared in the earlier work [2].

1. E. J. Cockayne, Chessboard domination problems, Discrete Math. 86 (1990), 13-20.

2. V. Raghavan and S. M. Venkatesan, On bounds for a covering problem, Inform. Process. Lett. 25 (1987), 281-284.

\section{Corrigendum added October 5th 2017}

Some incorrect attributions in the second paragraph of the second page of the paper are corrected in the next paragraph.

In 1863, C. F. de Jaenisch [22] considered the problems of finding the values of $\gamma\left(Q_{n}\right)$ and $i\left(Q_{n}\right)$, giving minimum dominating sets and minimum independent dominating sets up to $n=8$. His work was briefly summarized by W. W. Rouse Ball [23] in 1892, who considered several other questions about queen domination. In 1901, W. Ahrens [20, Chapter X] gave minimum dominating sets for $Q_{9}$, and in 1902 and 1903, K. von Szily $[24,25]$ gave minimum dominating sets of $Q_{n}$ for $10 \leq n \leq 13$ and $n=17$. De Jaenisch, Ahrens, and von Szily also worked extensively to find the number of different minimum dominating sets for each $n$, often giving lists with one set from each symmetry class. Many of these results were collected by Ahrens in the 1910 edition [21] of his book, and can also be found in its later editions.

A summary of established values and bounds for $\gamma\left(Q_{n}\right)$ and $i\left(Q_{n}\right)$ is given on pages 17 and 18 of the paper, in the form of seven lists. These lists should be disjoint, but the second and fourth lists both include 25, which should occur only in the second list. That is, $\gamma\left(Q_{25}\right)=13$. (This was shown in [26], and also can be deduced from inequality (2) of the paper and the first dominating set given in Section 5.)

\section{References}

[20] W. Ahrens, Mathematische Unterhaltungen und Spiele (B. G. Teubner, LeipzigBerlin, 1901).

[21] W. Ahrens, Mathematische Unterhaltungen und Spiele (B. G. Teubner, LeipzigBerlin, 1910).

[22] C. F. DE JAenisch, Applications de l'Analyse Mathématique au Jeu des Échecs, Appendix, pages $244 \mathrm{ff}$ (St. Petersburg, 1863). 
[23] W. W. Rouse Ball, Mathematical Recreations \& Essays, 3rd Edition (revision by H. S. M. Coxeter of the original 1892 edition), Chapter 6, Minimum pieces problem (Macmillan, London, 1939).

[24] K. von SzILy, Das Minimalproblem der Damen, Deutsche Schachzeitung, 57(1902), 326-328.

[25] K. von SzIly, Das Minimalproblem der Damen, Deutsche Schachzeitung, 58(1903), 65-68.

[26] W. D. Weakley, Domination in the queen's graph, in: Graph Theory, Combinatorics, and Algorithms, Vol. 2 (Kalamazoo, MI 1992), Y. Alavi and A. Schwenk (Editors), 1223-1232 (Wiley, New York, 1995). 